\title{
First-order Size Effects in the Mechanics of Miniaturized Components
}

\author{
J.P.M. Hoefnagels ${ }^{1, a}$, P.J.M. Janssen ${ }^{1,2}$, Th.H. de Keijser ${ }^{2}$, M.G.D. Geers ${ }^{1}$ \\ ${ }^{1}$ Eindhoven University of Technology, Department of Mechanical Engineering, P.O.Box 513, \\ $5600 \mathrm{MB}$, Eindhoven, The Netherlands
}

\author{
${ }^{2}$ Materials Innovation Institute (M2i), P.O.Box 5008, 2600GA, Delft, The Netherlands \\ a e-mail: j.p.m.hoefnagels@tue.nl, tel:+31-40-2475894, Fax:+31-40-2447355
}

\begin{abstract}
Keywords: Miniaturization, size effects, microstructure, experimental-numerical analysis, micromechanics.
\end{abstract}

\begin{abstract}
This work analyses those size effects that are encountered first upon downscaling, including grain boundary effects, free surface effects, grain statistics effects. The separate influence of first-order effects was carefully investigated from uniaxial tensile tests on high-purity aluminum specimens with a well-defined microstructure of through-thickness grains, whereby the total number of grains in the cross-section was reduced towards a single grain in a cross-section by, first, decreasing the film thickness and, second, for specimens with through-thickness grains decreasing the specimen width. In addition, 3D dislocation-field strain gradient plasticity simulations were employed to analyze the intrinsic size effects, using the grain size and texture as measured experimentally.

The work shows that for miniaturized structures with a limited number of columnar grains a unique Hall-Petch relation does not exist, even though a grain boundary effect, i.e. a decrease in stress level (at a given strain) for decreasing grain boundary area per unit volume, is clearly present. When the microstructure is kept constant upon miniaturization, the free surface per unit area increases causing the stress level of the structure to decrease, the effect of which increases towards a single grain in the cross-section. In addition, the work shows that grain statistics effects also contribute to observed weakening, due to insufficient compensation of local (weaker) material properties by the surrounding material (i.e. grains). Finally, grain statistics also significantly increase the statistical variation in mechanical properties for small-sized structures, an effect that is especially important for the reliability of miniature components. The separate influence of these first-order effects as well as their interplay are explained in terms of the movement of the dislocations upon plastic flow.
\end{abstract}

\section{Introduction}

Miniaturization is a general trend in the medical and microsystems industry, driving the design and manufacturing of smaller and smaller devices, structures, and parts. When the part's dimensions are decreased to the same order of magnitude as the material's characteristic microstructural length scales, significant changes in mechanical properties may occur, i.e. a so-called "size-effect". In mechanical loading, through e.g. forming, shaping, actuating and sensing (securing) of such materials, free surface and size effects are gaining importance and traditional continuum mechanics approaches thereby loose their validity and predictive nature. Also, if only a few grains are present across one of the dimensions of a component, the response to the applied forces may show great variation among components: hence, the reproducibility of the mechanical performance may become a problem. Overlooking the above effects in the industrial design of mechanics at small length scales may well lead to erroneous or too costly solutions.

This work analyses the seperate influence of some of those size effects that are encountered first upon downscaling from a larger size, including grain boundary effects, free surface effects, and grain statistics effects [1-10]. Upon miniaturizing a component (with constant microstructure), the total grain boundary area is inherently decreased, whereas the fraction of grains with a free surface is inherently increased. This give rise to a grain boundary and free surface effect. In addition, each 
grain with its specific orientation, size, shape, and connectivity to neighboring grains can be regarded as a unique sample from the bulk material's microstructure, particularly in top-down manufacturing. As the component's dimensions decrease, while the microstructure remains 'constant', a limited number of grains will be present inducing a sampling effect. Hence, the overall mechanical response of the component will be determined to an increasing extent by the mechanical properties of its individual grains, i.e. a so-called grain statistics effect may occur. Although limited evidence is available, it has been reported in literature that grain statistics effect results in an increased statistical variation of the stress level at a specific strain of the component, which may decrease the average stress level [8-10]. This article studies the above-mentioned first-order size effects and their interplay in more detail by following a coupled experimental numerical approach.

The experimental and numerical methodology is chosen such that the influence of other size effects can be excluded to the best of possibilities. For instance, high-purity aluminum sheet is selected to exclude the effect of second phase particles, while the FCC slip systems of Al are well understood and the high stacking fault energy hampers twin formation. To exclude undesired microstructural effects from the analysis, the specimen's microstructure (e.g., grain size, grain orientation) was characterized in detail via Orientation Imaging Microscopy. Furthermore, the mechanical behavior is characterized in uniaxial tension, because strain gradient effects can be neglected in first instance under macroscopically homogeneous deformation. The specimen and grain size dimensions (in experiments and simulations) were chosen such that, first, the number of grains in thickness was decreased from approximately three to one, i.e. columnar grains. Thereafter, the number of grains in the specimen width was also decreased towards one, i.e. a single grain in a cross-section. The relevance of this analysis is illustrated in Fig. 1, where a cross-section of a part of a free-standing metallic MEMS-component (aluminum-based RF-MEMS) is depicted. The presence of a limited number of grains (here in the width direction) or even a single grain (here in the thickness direction) is a practical and relevant issue for the engineering community.

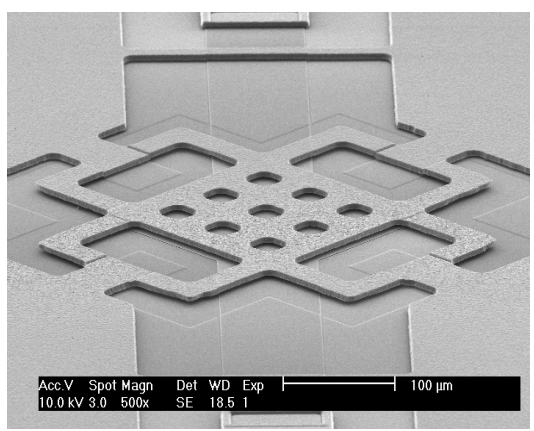

(a)

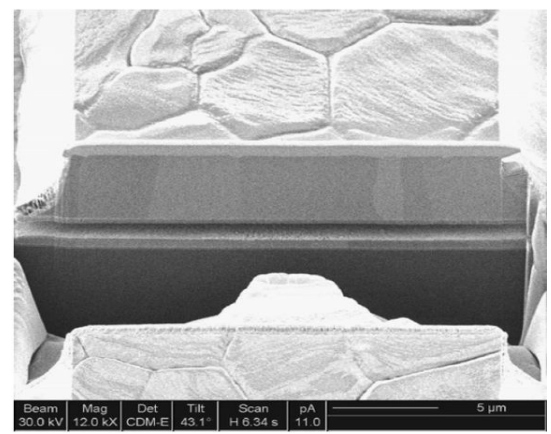

(b)

Fig. 1. (a) An aluminum-based MEMS capacitor and (b) a cross-section of an Al-based MEMScomponent showing it's through-thickness columnar grain structure (images courtesy of Philips).

\section{Experimental methodology}

Material selection and specimen types. For the experimental investigation, two sets of tensile specimens, set $\mathrm{I}_{\exp }$ and $\mathrm{I}_{\mathrm{exp}}$, have been prepared, starting from high-purity aluminum sheet material (99.999\%, Goodfellow). All tensile specimen types in both specimen sets have a well-defined, homogeneous, and reproducible cube texture and an initially very low dislocation density. The smallest thickness considered equals $95 \mu \mathrm{m}$, from which it can be safely concluded that all effects observed are most dominantly of the first-order type. Special care has been taken to minimize processing induced size effects [11], by using an elaborate specimen fabrication procedure [7,12].

Specimen Set $\mathrm{I}_{\exp }$ comprises of 7 different tensile specimen types that only differ in their thickness $(95-278 \mu \mathrm{m})$ and grain size $(99-379 \mu \mathrm{m})$. Note that all specimens have at most a few grains across the thickness. In order to study first-order size effects upon miniaturization, specimen 
set $\mathrm{I}_{\exp }$ includes microstructures ranging from multiple grains over the thickness to a single grain over the thickness. For a typical specimen with through-thickness grains, the microstructure and $\{100\}$ pole figure are shown in Fig. 2(a); microstructure image and pole figures of all 7 specimen types in set $\mathrm{I}_{\text {exp }}$, along with detailed information on the processing steps used, can be found in [7].

Specimens in set $\mathrm{II}_{\mathrm{exp}}$ all have the same homogeneous, reproducible microstructure of throughthickness pancake shaped grains with an average grain size of $800 \mu \mathrm{m}$ (area averaged, measured on the specimen surface) as well as a pronounced cube texture. These specimens also all have a thickness of $300 \mu \mathrm{m}$ and only differ in the width of the specimen, yielding a range of tensile specimens having from $\sim 1$ to $\sim 16$ columnar grains across the width. The pole figure of the microstructure of specimen set $\mathrm{II}_{\text {exp }}$ is shown in Fig. 2(b). More details are given in [12].

Uniaxial tensile experiments were performed at room temperature using a Kammrath\&Weiss 10 $\mathrm{kN}$ micro-loading stage equipped with a $100 \mathrm{~N}$ load cell. The displacements and strains were measured on the specimen surface by digital image correlation (e.g. [13]) (Aramis, www.gom.com). The tensile tests were displacement controlled and the strain rate was $5.0 \cdot 10^{-4} \mathrm{~s}^{-1}$. Engineering stresses, $\sigma_{e}$, and linear strains, $\varepsilon$, were determined from the measured forces and elongations. For more details about the complete experimental procedure the reader is referred to $[7,12]$.
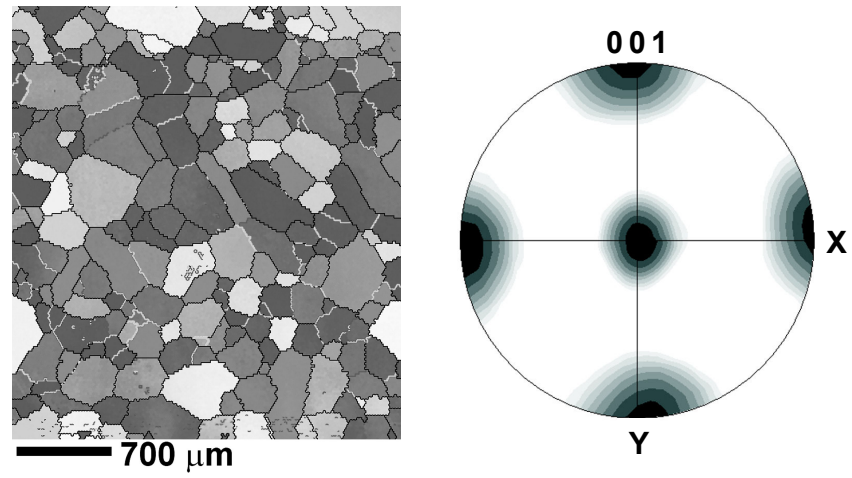

(a)

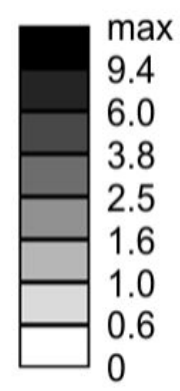

(b)

Fig. 2. (a) microstructure and $\{001\}$ pole figure of a typical specimen from specimen set $I_{\text {exp }}$ and (b) the $\{001\}$ pole figure of the specimens in set $I I_{\text {exp. }}$. The $x$-direction is parallel to the tensile direction. The grey-scale bar indicates the density levels for the pole figures (in arbitrary units). All data was obtained from orientation imaging microscopy.

\section{Numerical methodology: 3D dislocation-field strain gradient crystal plasticity model}

In order to investigate grain statistics effects numerically, a second-order strain gradient crystal plasticity model is used, which was initially developed by Evers et al. [14,15] and further extended by Bayley et al. [10,16]. In this model both the influence of geometrically stored dislocations (GNDs) and statistically stored dislocations (SSDs) are incorporated through the crystallographic slip resistance. For the present purpose all 12 slip systems of FCC crystals are modeled. The gradients of the GND field are used to determine the internal stress state that acts as a dislocation induced back stress. This model is well in line with other constitutive models, which incorporate strain gradient and dislocation phenomena and predict size dependence mechanical behavior, e.g. [17-20]. The extended model of Evers et al. proved suitable for the investigation of grain statistics effects in specimens with a limited number of grains [10]. Details on the internal stress formulation, the SSD and GND dislocation density evolution, the FEM implementation, and the solving procedure (of the highly non-linear and coupled equations) can be found in Refs. [10,14].

Using this model, first-order size effects are analyzed for tensile specimens with throughthickness grains and varying width. The specimens consist of square, pancake-shaped grains: the interior (bulk) grains have a length and width of $708 \mu \mathrm{m}$ and a height of $300 \mu \mathrm{m}$. To connect the simulations to the experiments, the grain area is taken equal to the (average) grain area of the 
pancake-shaped grains observed in the specimens of set $\mathrm{II}_{\text {exp }}$. The specimen length and thickness are $4.96 \mathrm{~mm}$ (7 grains in length) and $300 \mu \mathrm{m}$; the specimen structure is illustrated in Fig. 3 for a specimen with three full-size crystals in its cross-section (type h). Dislocations are able to escape from the specimen outer surfaces, i.e. the specimens have unconstrained free surfaces, and are blocked at the grain boundaries, i.e. the grain boundaries are impenetrable for dislocations.

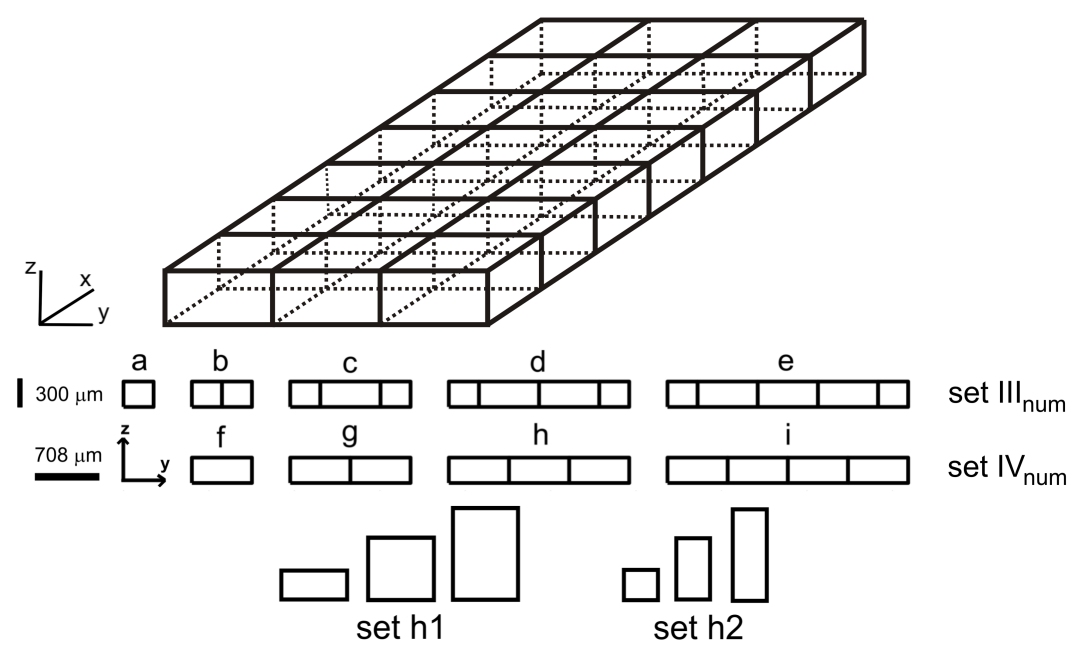

Fig 3. Three-dimensional specimen structure (shown here for specimen type $h$ ) with the $x$ direction parallel to the specimen length and tensile direction. The cross-sections in the yz-plane below show the different grain structures used in the simulations.

Three specimen sets are considered in the current analysis. Set $\mathrm{III}_{\text {num }}$ has half-sized edge grains with a width of $354 \mu \mathrm{m}$, representative for top-down manufacturing for which the edge grain size is on average half the bulk grain size. To assess the influence of these narrowed edge grains, the mechanical response of specimen set $\mathrm{III}_{\text {num }}$ is compared to specimen set $\mathrm{IV}_{\text {num }}$ with full-size edge grains, i.e. a width of $708 \mu \mathrm{m}$. The cross-sections of set $\mathrm{III}_{\text {num }}$ and $\mathrm{IV}_{\text {num }}$ are shown in Fig. 3. A third set of specimens, $V_{\text {num }}$, is used to test the influence of the grain shape. For this set, the lateral size (length and width) is $708 \mu \mathrm{m}$ or $354 \mu \mathrm{m}$ for set h1 and set h2, respectively, whereas the height, $t$, of the specimens is chosen either $300 \mu \mathrm{m}, 600 \mu \mathrm{m}$, or $900 \mu \mathrm{m}$.

The grain orientations were randomly sampled from measured orientations in the cube-textured Al specimens. In the FEM analysis, 27-node brick elements are used with a size of $177 \mu \mathrm{m}$ by 177 $\mu \mathrm{m}$ and a thickness of $100 \mu \mathrm{m}$. Consistent with the experimental work, material parameters of aluminum were used in the present analysis [8,21], except for the initial SSD density and the slip rate sensitivity, which are obtained directly from a experimental reference stress-strain curve [12].

\section{Results of the experimental-numerical analysis}

Grain boundary effect: grain size versus specimen dimensions. The investigation of the different first-order size effects and their interplay will start from the careful experimental analysis by Janssen et al. [7] of the grain size influence at a gradually reducing number of grains across the thickness in a tensile specimen. The most important results from Ref. [7] are shown in Fig. 4, concerning specimen set $\mathrm{I}_{\text {exp }}$. To assess if a Hall-Petch type relation $\left(\sigma=\sigma_{0}+k / \sqrt{d_{s}}\right)$, which would reflect an increased hardening upon grain refinement, holds for these kind of specimens with a few of grains over the cross-section, the true stress is plotted in Fig. 4(a) as a function of $1 / \sqrt{d_{s}}$, with $d_{s}$ the grain size measured on the specimen surface. The true stress clearly depends on the grain size, however, the specimens can be divided according to their film thickness, $t$, which is either $\sim 270 \mu \mathrm{m}$ or $\sim 100 \mu \mathrm{m}$. Both groups may obey separately a thickness-dependent Hall-Petch relation, though it is clear that no single straight line represents all data. Therefore, a single Hall-Petch relation looses its validity upon miniaturization, demonstrating a clear deviation from classical bulk behavior. 


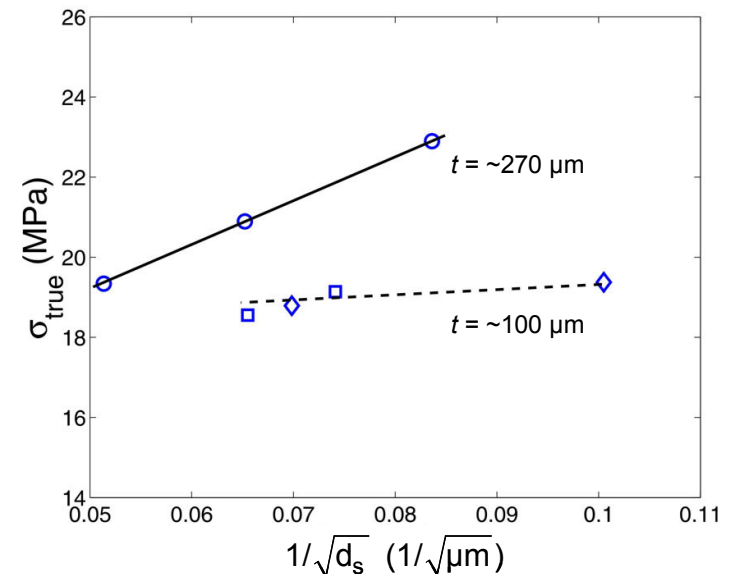

(a)

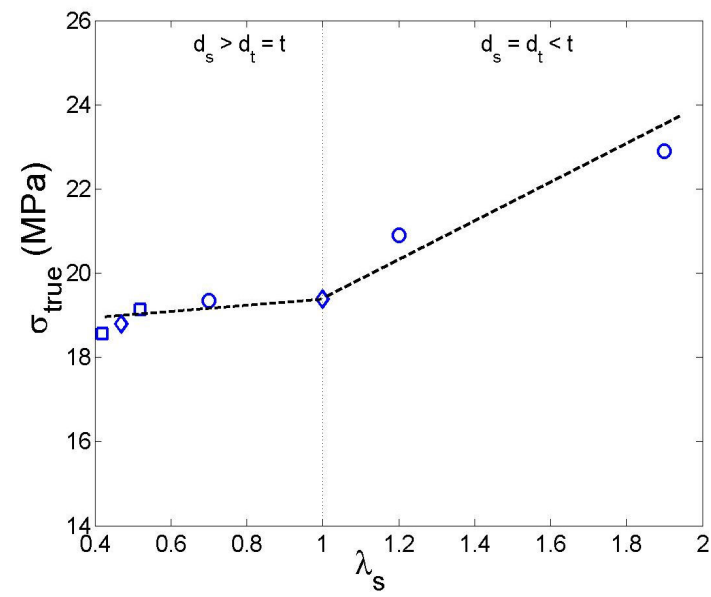

(b)

Fig 4. True stress at a logarithmic strain of $\varepsilon=0.1$ for the 7 specimen types in set $I_{\text {exp }}$ plotted as a function of (a) $1 / \sqrt{d_{s}}$ and (b) parameter $\lambda_{s}\left(\lambda_{s}=d_{s} / t\right)$. Figures adapted from [7].

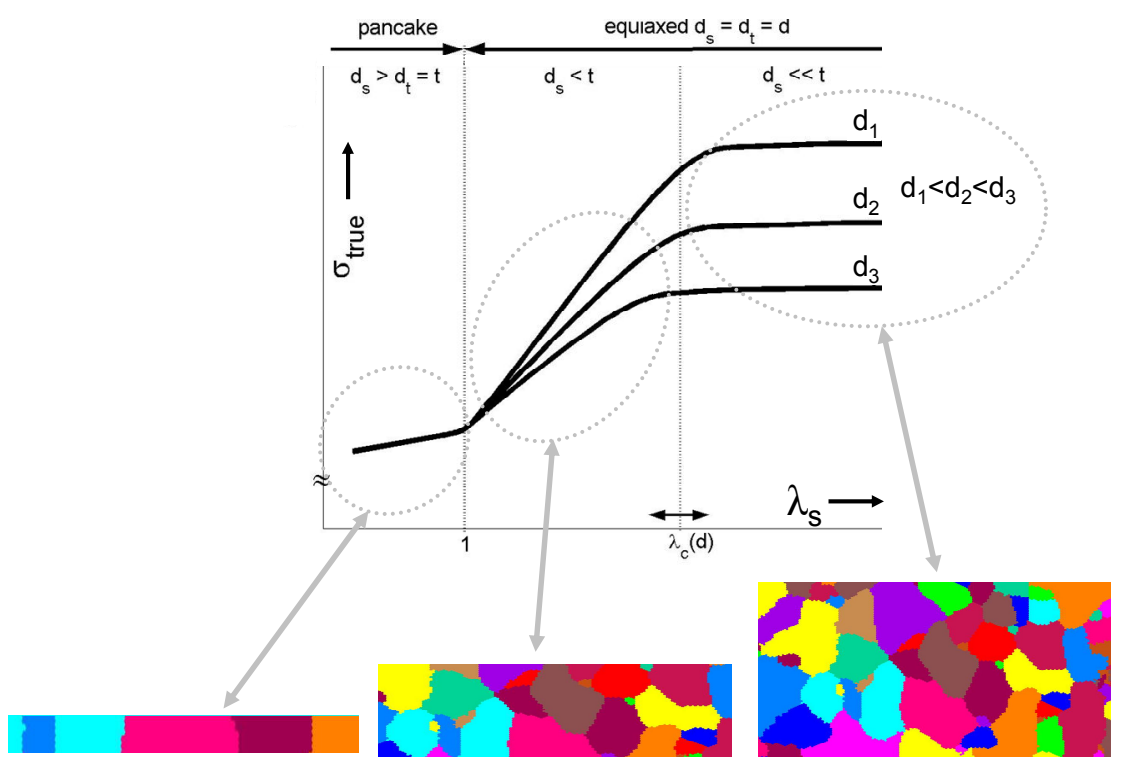

Fig 5. Schematic illustration of the dependence of the tensile strength on the grain size for tensile sheets with a limited number of grains across the thickness. Three different regimes can be identified, as illustrated by the cross-sectional microstructures below. Figure adapted from [7].

If we now plot the same data as a function of $\lambda_{\mathrm{s}}$, which is the ratio of the lateral grain size, $d_{s}$, over the film thickness, $t$, Fig. 4(b) shows that all data falls onto the same curve. Two regimes can be identified: (i) $1<\lambda_{\mathrm{s}}<3$ (i.e. $d_{t}=d_{s}<t$; where $d_{t}$ denotes the characteristic length of the grains in the thickness direction) and (ii) $\lambda_{\mathrm{s}} \leq 1$ (i.e. $d_{t}=t$ ). For $\lambda_{\mathrm{s}}>1$, a significant decrease in stress for decreasing $\lambda_{\mathrm{s}}$ is observed, whereas after $\lambda_{\mathrm{s}}$ has become less then one the decrease in stress with $\lambda_{\mathrm{s}}$ has reduced considerably. This difference in mechanical behavior can be explained by considering the microstructure in direct relation to the specimen dimensions. For $\lambda_{s}>1$, 'horizontal' grain boundaries are present in the specimens that make the material more resistant to deformation. For decreasing $\lambda_{\mathrm{s}}$, the density of horizontal grain boundaries decreases causing a significant decrease in stress. However, for $\lambda_{s} \leq 1$, only vertical' grain boundaries are left in the specimens. Upon further decrease of $\lambda_{s}$, the columnar grains change towards pancake-shaped grains, however, the overall grain boundary density only decreases slightly, causing the insensitivity of the stress to $\lambda_{\mathrm{s}}$.

Based on these results and in correspondence with prior findings in literature, the mechanical behavior on $\lambda_{\mathrm{s}}$ upon miniaturization was summarized in a general representation (for the case that 
strain-gradient effects can be ignored and dislocations are not significantly obstructed in their escape from the surface), as shown in Fig. 5. Three regions were distinguished:

- $\lambda_{\mathrm{s}}<1$ and $d_{s}>d_{t}=t$ : slight increase of $\sigma_{\text {true }}$ with increasing $\lambda_{s}$ due to an increase of the volume fraction of hard vertical grain boundary regions. The stress is only dependent on $\lambda_{\mathrm{s}}$ and not on the absolute thickness or average grain size.

- $1<\lambda_{\mathrm{s}}<\lambda_{\mathrm{c}}$ and $d_{s}=d_{t}<t$ : strong increase of $\sigma_{\text {true }}$ with increasing $\lambda_{\mathrm{s}}$ due to the increase of the volume fraction of hard horizontal grain boundary regions.

- $\lambda_{\mathrm{s}}>\lambda_{\mathrm{c}}$ and $d_{s}=d_{t}<<t$ : reducing increase of $\sigma_{\text {true }}$ with increasing $\lambda_{\mathrm{s}}$ due to the decrease of the volume fraction of crystals with a free surface.

For $\lambda_{\mathrm{s}}<1$, there is a single curve independent of $d$ and $t$, whereas for $\lambda_{\mathrm{s}}>1$ there is a dependence on the grain size (Hall-Petch type effect). For $\lambda_{\mathrm{s}}>>\lambda_{\mathrm{c}}$, there is no longer any free surface effect noticeable, therefore, the slope of the flow stress approaches zero.

In addition, the slight increase in stress with increasing $\lambda_{\mathrm{s}}$ for specimens with through-thickness grains (the regime of $\lambda_{\mathrm{s}}<1$ in Fig. 4(b)) was explained in [7] on the basis of a model in which the specimen is considered as a two-dimensional arrangement of crystals (each with two free surfaces) that effectively consist of a softer central part of relatively free dislocation movement and harder regions nearby the grain boundaries where dislocation movement is severely restricted. Based on this model of columnar-grained specimens, a hypothesis was presented in [12] for the change in material behavior associated with the transition from pancake-shaped grains to needle-shaped grains, i.e. going from $\left(\lambda_{\mathrm{s}}<1\right.$ and $\left.d_{t}=t\right)$ to $\left(\lambda_{\mathrm{s}}>1\right.$ and $\left.d_{t}=t\right)$. This hypothesis is schematically presented in Fig. 6(a). Basically, for increasing $\lambda_{s}$ the grains become narrower, resulting in an increased influence of the harder grain boundary regions, yielding a gradual increase of $\lambda_{\mathrm{s}}$. This trend will continue up to the point where the harder regions nearby the grain boundaries will overlap each other, at which point a sudden increase in $\sigma_{\text {true }}$ is to be expected.

Using new 3D dislocation-field strain gradient crystal plasticity simulations, the validity of the hypothesis can now be verified by simulating specimens with different lateral grain size (either 708 or $354 \mu \mathrm{m})$ and different grain height $(300,600$, or $900 \mu \mathrm{m})$, see specimen set h1 and h2 in Fig. 3. For the grains, both edges have an obstructed dislocation motion, which partially takes the grain boundary effect into account [12], while the dislocations are free to escape from the top and bottom surfaces. The simulated engineering yield stresses as a function of $\lambda_{\mathrm{s}}$ are shown in Fig. $6(\mathrm{~b})$.

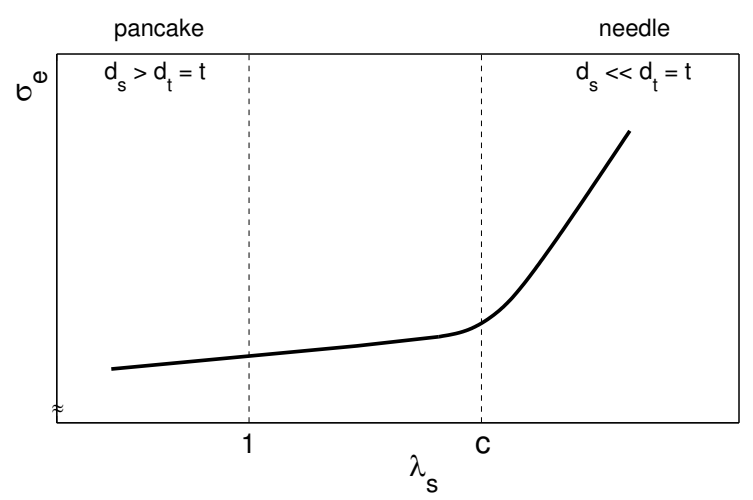

(a)

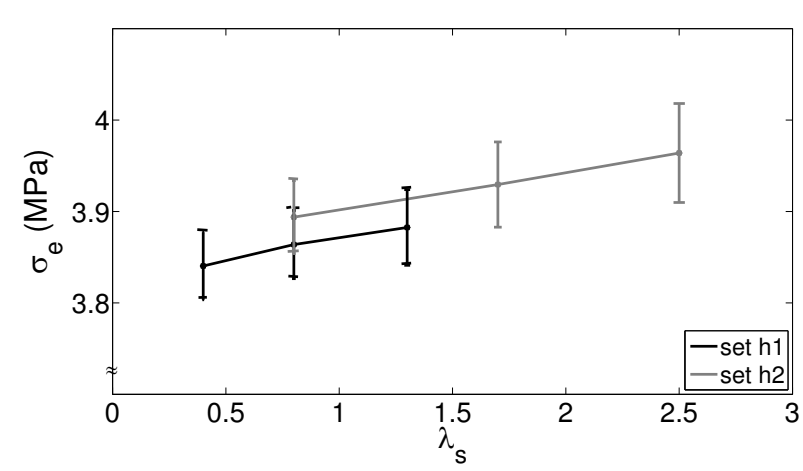

(b)

Fig 6. Hypothesis reported in [12] regarding the mechanical behavior for specimens with columnar grains as a function of $\lambda_{s}$ and (b) the engineering yield stress versus $\lambda_{s}$ from simulations of specimen set $V_{\text {num }}$ [22]. See Fig. 3 for grain shape of specimen set h1 and h2.

Both for specimen set h1 and h2, the slope found in Fig. 6(b) agrees with the experimental slope in Fig. 4(b) for $\lambda_{s}<1$ within experimental uncertainty [22]. This confirms the conclusion that the flow stress principally depends on $\lambda_{\mathrm{s}}$, and only indirectly on thickness or grain size. Moreover, the results of set $\mathrm{h} 1$ and set $\mathrm{h} 2$ show only a small increase in stress with increasing $\lambda_{\mathrm{s}}$, which agrees with the (postulated) behavior indicated in Fig. 6(a) for $\lambda_{\mathrm{s}}<1$. These findings are also supported by 
the results presented in the work of [23] for thin films with a thickness smaller than $5 \mu \mathrm{m}$. As was already concluded in [7], for these specimens with sufficiently flat grains, the central part of the grains can deform and rotate more easily than the hardened zones around the grain boundaries.

Miniaturization: grain boundary, free surface, and grain statistics effects. The measured stress-strain responses of the 2.2 and $9.0 \mathrm{~mm}$ wide specimens of set $\mathrm{II}_{\mathrm{exp}}$ are shown in Fig. 7. While the trends in the average stress may appear similar at first instance, clearly, the statistical variation is significantly larger for the narrow specimens. The smaller the number of grains in a cross-section, the less accurately the (average) microstructure of the base material is represented and the more the local microstructure dominates the overall mechanical response, resulting in a grain statistics effect. Additional insight in the statistical variation due to grain statistics is obtained by considering the ratio of the standard deviations of the stress of the narrow and wide specimens from Fig. 7, yielding a ratio that is somewhat below 2. This ratio is better reproduced by a sampling of the number of columnar grains in the cross-section when taking into account half-sized grains at the edges, than by a sampling of some (unknown) material property for which its random variation scales inversely linear with the specimen width. This again suggests that the observed statistical variation in Fig. 7 is caused by a sampling of the bulk material's microstructure, i.e. a grain statistics effect.

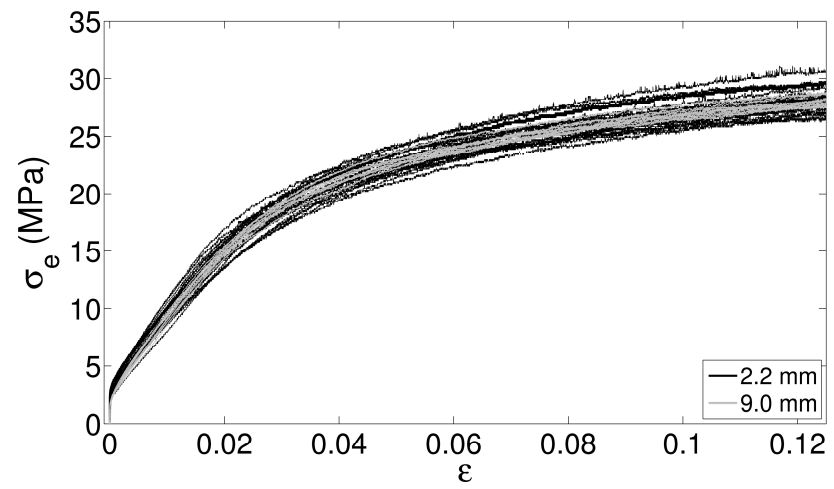

Fig. 7. Stress-strain curves for all specimens of set $I I_{\text {exp. }}$.

The increasing influence of the local material properties on the mechanical behavior of specimens with a smaller size is most clearly demonstrated by the evolution of the strain fields of the $2.2 \mathrm{~mm}$ and $9.0 \mathrm{~mm}$ wide specimens, respectively, as shown in Fig. 8. In the narrow specimens, localized narrow slip bands of equal strain develop across nearly the full specimen width already ate early stages of deformation (Fig. 8(a)-8(c)). These zones remain active during the complete tensile test. In the wide specimens, areas of localized deformation of a roughly equal size are also visible, however, only close to fracture several of these zones coalesce to span the full specimen width (Fig. 81). From the inhomogeneities in the strain fields, it is obvious that the deformation localizes at weak grains in the specimen, which are much less constrained in the width direction for the narrow specimens. For the wide specimen, weak grains are constrained in deformation by neighboring grains in the same cross-section, postponing localization across the full specimen width.

The averaged stress-strain responses of the 2.2 and $9.0 \mathrm{~mm}$ wide specimens of set $\mathrm{II}_{\text {exp }}$, obtained from the data in Fig. 7, do not shown a significant difference (not shown here). Apparently, the still relatively large number of grains in the cross-section (4.4 versus 15.7 , respectively), combined with a strong (cube) texture (which decreases the influence of the grain statistics effect), does not result in a distinct influence of the intrinsic size effects (grain boundary effect, free surface effect, and grain statistics effect) on the average mechanical response. Therefore, additional tensile experiments have been performed on specimens with a much larger average grain size of $3.3 \mathrm{~mm}$ and a random texture, having a width of 2.0 and $9.0 \mathrm{~mm}(\sim 2$ and $\sim 5$ grains across the width, respectively). Preliminary results show a strong decrease in the averaged flow stress with decreasing width [12]. However, due to the complexity of these sensitive measurements, it remains difficult to sharply discriminate between the different size effects on the basis of the experiments. To overcome 
this seemingly fundamental problem, 3D dislocation-field strain gradient crystal plasticity simulations have been conducted on specimens of varying width, with the other dimensions taken equal to specimen set $\mathrm{II}_{\text {exp }}$ and using grain orientations that were randomly sampled from the orientations present in the cube-textured $\mathrm{Al}$ specimens used in the experiments of set $\mathrm{II}_{\text {exp }}$.

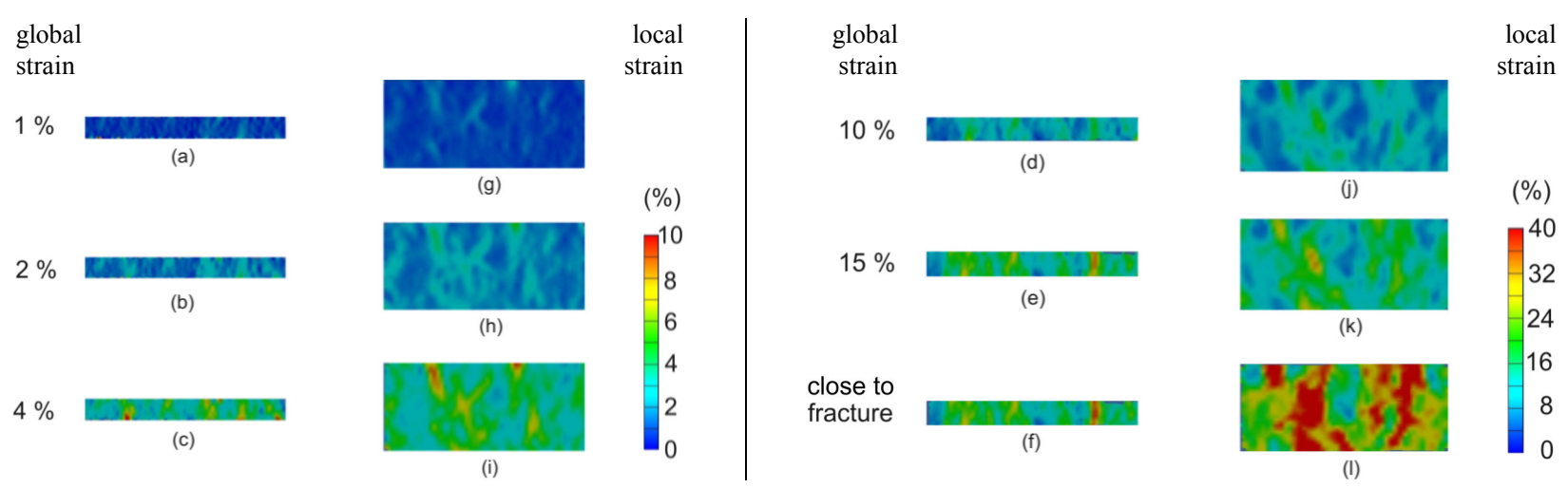

Fig. 8. Strain field evolution measured by digital image correlation on the surface of a $2.2 \mathrm{~mm}$ [subfigures (a)-(f)] and $9.0 \mathrm{~mm}$ wide specimen [subfigures $(g)-(l)]$ both of set $I I_{\text {exp. }}$. The length of the analyzed area is about $20 \mathrm{~mm}$.

The engineering yield stresses $\left(\sigma_{\mathrm{y}}{ }^{\mathrm{e}}\right)$, i.e. the macroscopic stress level at a uniaxial tensile strain of $0.2 \%$, or $\mathrm{Rp}_{0.2 \%}$, are shown in Fig. 9. In Fig. 9(a), the results for all individual simulations are presented, which shows the (large) variation due to the grain statistics effect. The average stresses are shown in Fig. 9(b), error bars indicating the standard deviation of the average. From these results, the influence of grain boundaries, free surfaces, and grain statistics effects are assessed:

Figure 9(b) shows that the engineering yield stress decreases with decreasing specimen width for both specimen sets (type (e)-(a) and type (i)-(f)). This decrease is most pronounced going from a single to no grain boundary within a cross-section. For decreasing grain boundary area per unit volume, the mobility of dislocations increases and the constraints on the grain rotation and deformation imposed by neighboring grains decrease, resulting in a weaker response. In the range of type (e)-(b) (set $\mathrm{III}_{\text {num }}$ ), a grain boundary effect is less relevant, since the grain boundary area per unit volume is the same for these specimens. However, for decreasing specimen dimensions the contribution of the free surfaces (of the edge grains) increases, which also leads to weakening.

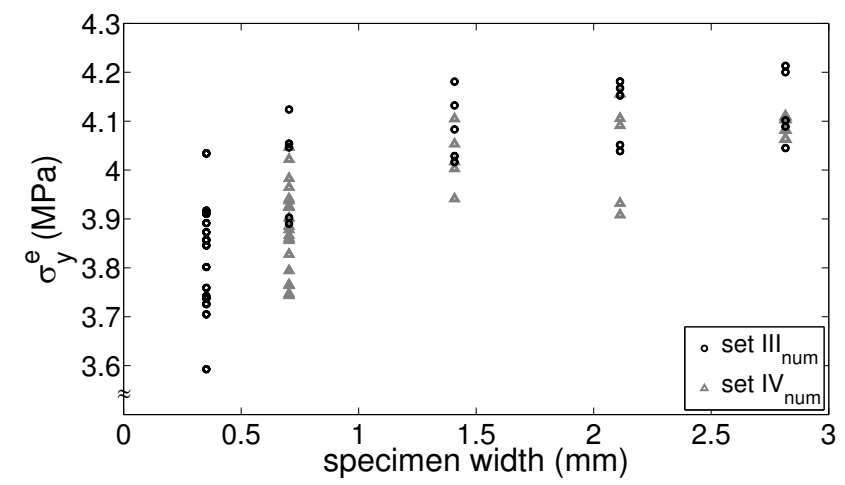

(a)

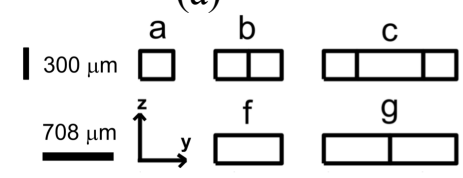

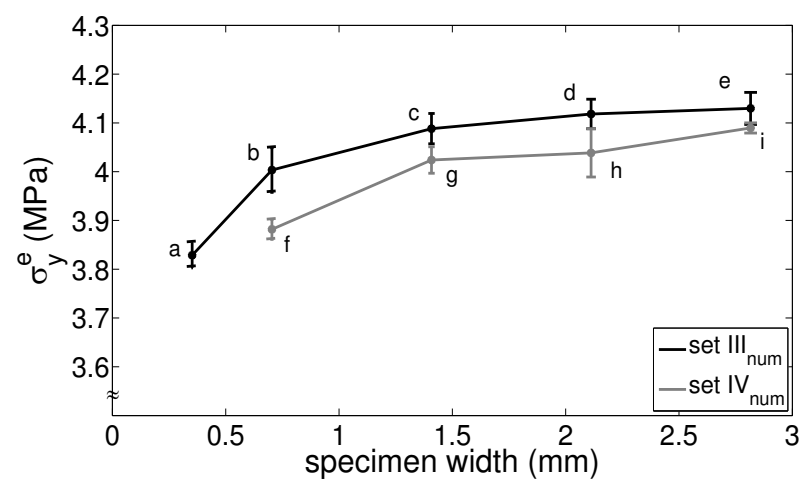

(b)
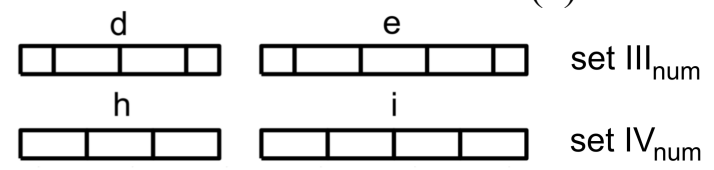

Fig 9. Simulation results of the engineering yield stress $\left(R p_{0.2 \%}\right)$ for specimens of varying width and edge grain size: (a) all individual results and (b) averages per specimen type with the error bars indicating the $68 \%$ confidence interval of the average. In addition, the crosssections of the used specimen types are shown below. 
It is further noted from Fig. 9(b) that the grain boundary area per unit volume for the specimens of set $\mathrm{III}_{\text {num }}\left(\right.$ types (b)-(e)) is higher than that for the considered specimens of set $\mathrm{IV}_{\text {num }}($ types $(\mathrm{g})-(\mathrm{i})$ ). Conversely, the grain boundary area per unit volume increases with increasing specimen width for set $I_{\text {num }}$ towards the value for set III $_{\text {num. }}$. Therefore, the average stress level for the specimens of set $\mathrm{IV}_{\text {num }}$ is lower at equal specimen width, but converges towards the stress level of the specimens of set $\mathrm{III}_{\text {num }}$ for increasing specimen width. The strengthening effect of a single vertical grain boundary is emphasized by comparing the stress levels for specimens with two half-size grains across the specimen width (type (b)) and with a single full-size grain across the specimen width (type (f)). Although neglected at first instance, the grain statistics effect may also have an influence on the average mechanical properties of specimens of a certain type. For instance, the weakening upon decreasing specimen dimensions shown in Fig. 9(b), may also result (in part) from grain statistics due to weak grains dominating the mechanical behavior. In order to study the separate contribution of grain statistics effects on the average mechanical behavior, the influence of grain statistics simulations is artificially neutralized in the simulations. These simulations without grain statistics effect [24] show that, although there is still a decrease in stress level for decreasing specimen width, this decrease is significantly less then seen in Fig. 9. This proves that grain statistics contributes to a non-negligible decrease in the average strength of a component upon miniaturization.

\section{Conclusions}

This work investigated the following intrinsic (first-order) size effects: grain boundary effects, free surface effects, and grain statistics effects. To this cause, specimens with a well-defined microstructure and specific geometries (relative to the microstructural size) were carefully prepared. The mechanical behavior was carefully measured under uniaxial tension and complemented with numerical tensile simulations (in the yielding regime). The main conclusions of the research are:

- For miniaturized components with a limited number of grains in one of their dimensions a unique Hall-Petch relation does not exist, even though a grain boundary effect is clearly present.

- Decreasing the component size at constant microstructure, results in an increasing impact of free surface effects on the mechanical properties. The stress level (at a given strain) decreases for increasing volume fraction of grains with a free surface, because these are less constrained in deformation and rotation as their counterparts in the bulk. Simultaneously, decreasing the component size at constant microstructure results in a decrease of the grain boundary area per unit volume as well, which also yields a decrease in the stress level. The free surface effect and grain boundary effect seem intrinsically coupled in experiments and engineering practice.

- For components with a single grain across the thickness and a considerable number of grains in the other dimensions, the stress level depends primarily on the ratio of the thickness and grain size. The stress level decreases only slightly with decreasing ratio. This is explained by a model in which the central parts of the grains can deform and rotate more freely due to the absence of grain boundaries parallel to the component top and bottom surfaces.

- Grain statistics effects lead to a significant increase in statistical variation in the (mechanical) properties and also contribute to a non-negligible decrease in average stress level of small-sized components: for a decreasing number of grains in a cross-section, weaker local zones will become increasingly dominant in controlling the overall mechanical properties.

\section{Acknowledgements}

The authors greatly acknowledge Laurent Duchêne at the Department of Mechanics of materials and Structures of the University of Liège for his implementation of the strain-gradient plasticity model in FEAP. This research was carried out under the project number MC2.02114 in the framework of the Research Program of the Materials innovation institute M2i (www.m2i.nl), the former Netherlands Institute for Metals Research. 


\section{References}

[1] E. Arzt: Acta Materialia Vol. 46 (1998), p. 5611-5626

[2] S. Miyazaki, K. Shibata, and H. Fujita: Acta Metallurgica Vol. 27 (1978), p. 855-862

[3] S. Miyazaki, K. Shibata and H. Fujita: Acta Metall. Vol. 27 (1979), p. 855-862

[4] R.T.A. Kals: Fundamentals on the miniaturization of sheet metal working processes (Ph.D. thesis, Erlangen-Nürnberg, Germany 1998).

[5] R. Kals and R. Eckstein: Journal of Materials Processing Technology Vol. 103 (2000), p. 95-101

[6] E. Nakamachi, K. Hiraiwa, K.H. Morimoto, and M. Harimoto: International Journal of Plasticity Vol. 16 (2000), p. 1419-1441

[7] P.J.M. Janssen, TH.H de Keijser and M.G.D. Geers: Materials Science and Engineering A Vol. 419 (2006), p. 238-248

[8] T. Fülöp, W.A.M Brekelmans and M.G.D. Geers: Journal of Materials Processing Technology Vol. 174 (2006), p. 233-238

[9] M. Henning and H. Vehoff: Materials Science and Engineering A Vol. $452-453$ (2007), p. 602-613

[10] C.J. Bayley, W.A.M. Brekelmans and M.G.D. Geers: Philosophical Magazine A Vol. 87 (2007), p. $1361-1378$

[11] P.J.M. Janssen, J.P.M. Hoefnagels, Th.H. de Keijser and M.G.D. Geers: Journal of the Mechanics and Physics of Solids (2007), in press.

[12] P.J.M. Janssen: First-order size effects in the mechanics of miniaturised components (Ph.D. thesis, chapter 5, Eindhoven University of Technology, The Netherlands 2007)

[13] T.C. Chu, W.F. Ranson, M.A. Sutton and W.H. Petters: Experimental Mechanics Vol. 25 (1985), p. 232-244

[14] L.P. Evers, W.A.M. Brekelmans and M.G.D. Geers: Journal of the Mechanics and Physics of Solids Vol. 52 (2004), p. 2379-2401

[15] L.P. Evers, W.A.M. Brekelmans and M.G.D. Geers: International Journal of Solids and Structures Vol. 41 (2004), p. 5209-5230

[16] C.J. Bayley, W.A.M. Brekelmans and M.G.D. Geers: International Journal of Solids and Structures Vol. 43 (2006), p. 7268-7286

[17] M.E. Gurtin: Journal of the Mechanics and Physics of Solids Vol. 50 (2002), p. 5-32

[18] M.E. Zaiser and E.C. Aifantis: Scripta Materialia Vol. 48 (2003), p. 133-139

[19] G.Z. Voyiadjis, R.K. Abu Al-Rub: International Journal of Solids and Structures Vol. 42 (2005), p. 3998-4029

[20] P. Dredriksson and P. Gudmundson: International Journal of Plasticity Vol. 21 (2005), p. 1834-1854

[21] L.P. Evers: Strain Gradient Crystal Plasticity based on Dislocation Densities (Ph.D. thesis, Eindhoven University of Technology, The Netherlands 2003)

[22] In the tensile experiments the stress level is compared at a somewhat later stage of deformation (strain of 0.1 ) in order to rule out a residual processing induced size effect which is mainly active at lower strains [12], however, for the simulations the stress level is compared at the engineering yield stress at $0.2 \%$ strain, $R p_{0.2 \%}$, in order to save computation time.

[23] L. Nicola, Y. Xiang, J.J. Vlassak, E. Van der Giessen, A. Needleman: Journal of the Mechanics and Physics of Solids Vol. 54 (2006), p. 2089-2110.

[24] P.J.M. Janssen, J.P.M. Hoefnagels, Th.H. de Keijser, and M.G.D. Geers: to be published. 